University of Wollongong

Research Online

Faculty of Science, Medicine and Health -

Papers: part A

Faculty of Science, Medicine and Health

$1-1-2016$

\title{
Effects of physical exercise on health and well-being of individuals living with a dementia in nursing homes: a systematic review
}

Lindsey Brett

University of Wollongong, Ikb267@uowmail.edu.au

Victoria Traynor

University of Wollongong, vtraynor@uow.edu.au

Paul J. Stapley

University of Wollongong, pstapley@uow.edu.au

Follow this and additional works at: https://ro.uow.edu.au/smhpapers

Part of the Medicine and Health Sciences Commons, and the Social and Behavioral Sciences

\section{Commons}

\section{Recommended Citation}

Brett, Lindsey; Traynor, Victoria; and Stapley, Paul J., "Effects of physical exercise on health and well-being of individuals living with a dementia in nursing homes: a systematic review" (2016). Faculty of Science, Medicine and Health - Papers: part A. 3550.

https://ro.uow.edu.au/smhpapers/3550

Research Online is the open access institutional repository for the University of Wollongong. For further information contact the UOW Library: research-pubs@uow.edu.au 


\title{
Effects of physical exercise on health and well-being of individuals living with a dementia in nursing homes: a systematic review
}

\begin{abstract}
Background Physical exercise interventions have benefits for older individuals and improve the health and well-being of individuals living with a dementia, specifically those living in nursing homes. Purpose Report evidence from randomized controlled trials and cluster randomized control trials that evaluated the effects of physical exercise interventions on individuals living with a dementia in nursing homes. Data sources Web of Science, Scopus, Science Direct, Academic Search Complete, Proquest Central, British Medical Journal Database, PubMed, Cochrane Library, PEDro, Informit, Informa, and Nursing Consult were searched for relevant clinical trials and snowballing of recommended studies. Study selection One reviewer screened articles on inclusion criteria and identified relevant studies. Data extraction Data extraction was performed by 1 reviewer and checked by second and third reviewers. Two authors assessed the methodological quality and risk of bias of the relevant studies. Data synthesis Twelve study populations consisting of individuals living with a dementia in nursing homes were included $(n=901)$. Different types of physical exercises were undertaken: multimodal $(n=6)$, walking $(n=5)$, music and movement $(n=2)$, and hand exercises $(n=1)$. The parameters of the interventions varied across the studies. Most of the studies reported significant positive effects of physical exercise on cognition, agitation, mood, mobility, and functional ability for individuals living with dementia in nursing homes. Limitations The main limitations were the heterogeneity of design, small samples, and short interventions. Conclusions There is emerging evidence that physical exercise significantly benefits individuals living with a dementia in nursing homes. Higher quality research is required adopting more rigorous methods, including longer interventions and larger samples to determine optimum parameters of the physical exercise interventions evaluated.

\section{Disciplines}

Medicine and Health Sciences | Social and Behavioral Sciences

\section{Publication Details}

Brett, L., Traynor, V. \& Stapley, P. (2016). Effects of physical exercise on health and well-being of individuals living with a dementia in nursing homes: a systematic review. Journal of the American Medical Directors Association (JAMDA): long-term care: management, applied research and clinical issues, 17 (2), 104-116.
\end{abstract}




\section{Effects of Physical Exercise on Health and Well-Being of Individuals Living With a Dementia in Nursing Homes: A Systematic Review}

Lindsey Brett BSc Hons *, Victoria Traynor PhD, BSc Hons, Paul Stapley PhD, MSc, BA Hons

Faculty of Science Medicine and Health, University of Wollongong, New South Wales, Wollongong, Australia

\section{Abstract}

Background. Physical exercise interventions have benefits for older individuals and improve the health and well being of individuals living with a dementia, specifically those living in nursing homes.

Purpose. Report evidence from randomized controlled trials and cluster randomized control trials that evaluated the effects of physical exercise interventions on individuals living with a dementia in nursing homes.

Data sources. Web of Science, Scopus, Science Direct, Academic Search Complete, Proquest Central, British Medical Journal Database, PubMed, Cochrane Library, PEDro, Informit, Informa and Nursing Consult were searched for relevant clinical trials and snowballing of recommended studies.

Study selection. One reviewer screened articles on inclusion criteria and identified relevant studies.

Data extraction. Data extraction was performed by one reviewer and checked by second and third reviewers. Two authors assessed the methodological quality and risk of bias of the relevant studies.

Data synthesis. Twelve study populations consisting of individuals living with a dementia in nursing homes were included ( $n=901)$. Different types of physical exercises were undertaken: multimodal $(n=6)$, walking $(n=5)$, music and movement $(n=2)$ and hand exercises $(n=1)$. The parameters of the interventions varied across the studies. Most of the studies reported significant positive effects of physical exercise on cognition, agitation, mood, mobility and functional ability for individuals living with dementia in nursing homes.

Limitations. The main limitations were the heterogeneity of design, small samples and short interventions.

Conclusions. There is emerging evidence that physical exercise significantly benefits individuals living with a dementia in nursing homes. Higher quality research is required adopting more rigorous methods, including longer interventions and larger samples to determine optimum parameters of the physical exercise interventions evaluated.

Keywords: exercise; physical activity; dementia; Alzheimer's; nursing home 


\section{Introduction}

As global fertility rates decline and life expectancy rises, the ageing population increases. In later life, the incidence of chronic conditions increases and an associated rise in the prevalence of age related morbidities, such as dementia. Worldwide, there are 35.6 million individuals living with a dementia and 7.7 million new cases are diagnosed each year ${ }^{(1)}$. The most common form of dementia is Alzheimer's Disease $(60-70 \%$ of cases) and other forms of dementia include vascular, Lewy bodies, frontotemporal, Parkinson's', multi infarct and mixed $^{(1)}$.

Most individuals living with dementia have regular contact with healthcare services. The overall aim of healthcare services for individuals living with dementia is to optimize their health and well being and that of their family carers, including treatment of the associated behavioral, psychological and physical symptoms of dementia $^{(1)}$. Dementia care services implement a range of strategies, such as psychosocial activities, behavior strategies, sensory stimulation, medication and physical exercise, to achieve their goals of improving the health and well being of individuals living with a dementia ${ }^{(2)}$. We know that physical exercise is beneficial for healthy older individuals by improving mobility, physical function, cognition and mood and preventing falls ${ }^{(3,4)}$. A Cochrane review reported that physical exercise significantly effects individuals with a dementia living in various settings but further research was needed to explain the specific effects and what type of physical exercise is most beneficial ${ }^{(5)}$.

Thirty percent of individuals with a dementia live in nursing homes due to the disabling effects and increased dependency associated with dementia ${ }^{(6)}$. A nursing home is an accommodation service in which an individual is provided with a high level of nursing and/or personal care, including care staf who provide these nursing and personal care needs as well as meals, cleaning and furnishings and equipment for the provision of that care ${ }^{(7)}$. Demands for nursing homes will increase as the prevalence of dementia continues to rise ${ }^{(8)}$. In nursing homes, care services and interventions are provided by a range of practitioners, including nurses, Occupational Therapists, Diversional Therapists and Physical Therapists. The main focus of the Physical Therapist is to direct the implementation of physical exercise strategies which improves and maintains range of movement, strength, balance, mobility and functional ability in individuals and contribute to improved quality of life for individuals living with a dementia in nursing homes. Physical exercise is defined as physical activity that is 
planned, structured, repetitive and has a final or intermediate objective of improving or maintaining physical fitness $^{(3)}$. It is important to know what type of physical exercise best suits this population to ensure the work of physical therapists is evidence based and engages individuals living with a dementia physically and mentally ${ }^{(9)}$. The purpose of this systematic review was to evaluate evidence from randomized controlled trials (RCTs) and cluster RCTs measuring the effects of physical exercise on the health and well being of individuals living with a dementia in nursing homes. The influence of the studies on future research and clinical practice was also considered.

\section{Methods}

This systematic review was informed by the Preferred Reporting Items for Systematic Reviews and Meta Analyses (PRISMA) Statement ${ }^{(10)}$.

\section{Data searches and study selection}

A comprehensive literature search was undertaken using a range of databases to retrieve relevant studies for the review: Web of Science, Scopus, ScienceDirect, Academic Search Complete, Proquest Central, British Medical Journal Database, PubMed, Cochrane Library, PEDro, Informit, Informa and Nursing Consult. The search terms used were: for population: "Dementia", "Alzheimer's"; for intervention: "Exercise", "Physical activity", "Physical intervention", "Physiotherapy", "Physical therapy", "Walking"; for setting: "Nursing home", "Residential accommodation", "Aged care facility", "Long term care", "Care home", "Rest home"; for outcome: "Physical function", "Healthcare outcome measure", "Behaviors", "Agitation". In addition, snowballing was used to locate additional references.

The titles and abstracts of all studies found in the database searches and from snowballing were screened and duplicates and irrelevant studies excluded. From the remaining studies, the full text was read to identify relevant information and checked against the inclusion criteria. Studies were eligible if they: (1) involved participants diagnosed with a dementia; (2) used a physical exercise intervention; (3) set in a nursing home; (4) were a RCT or cluster RCT; (5) published in English. The references of review papers identified were also checked for additional relevant studies that could have been missed in the database searches. 


\section{Methodological quality assessment}

The quality of the studies was critically appraised using the Joanna Briggs Institute Critical Appraisal Tool: Meta Analysis of Statistics Assessment and Review Instrument (MAStARI) critical appraisal tool for randomized control/pseudo randomized control trials ${ }^{(11)}$. Ten items were considered, they evaluated the randomization process, blinding, intention to treat analysis, homogeneity of groups, outcome measures and statistical analysis (Table 1). Each item was either answered 'yes' or 'no'. When there was insufficient information to answer the question or it was unclear 'no' was recorded. After completing the evaluation of a paper, it was given a score out of 10 and the score increased with higher methodological quality. Two reviewers score all selected studies and differences between reviewers are discussed and an agreement reached on the final score allocated to the study and if agreement cannot be met a third reviewer scores the studies. A cutoff value of the mean score minus one Standard Deviation is used to ensure only high quality studies are included in the review.

\section{Data extraction and analysis}

Data extraction was completed by one reviewer using a standardized extraction form. This was checked by second and third reviewers and any obscurities discussed to avoid potential errors or misinterpretation of results. A meta-analysis was not possible due to the large variability between the studies: physical exercise intervention, parameters applied, outcome measures, who conducted the intervention and type of dementia among the participants (not specified in majority of the studies).

\section{Results}

A PRISMA Consort Statement summarized the outcomes of the literature search (Figure 1). Screening the titles and abstracts found 1,722 (94\%) irrelevant and duplicated studies. Full texts of the remaining 102 studies were read and the inclusion criteria applied: 15 eligible studies of which three were excluded due to low methodological quality. Twelve studies were included in the systematic review: $11 \mathrm{RCTs}^{(12-24)}$ and one cluster RCTs ${ }^{(25)}$, of which one RCT had generated three separate publications. Williams C (2015 pers. comm., 16 July) and Roach K (2015 pers. comm., 22 July) verified through email communications that each publication was drawn from the same sample $e^{(14,22,23)}$. 


\section{Methodological quality}

The methodological quality of the studies was assessed using the MAStARI critical appraisal tool for randomized/pseudo randomized control trials (from the Joanna Briggs Institute Critical Appraisal Tools) (Table $1)^{(11)}$. Both reviewers were able to agree on the scores for all studies without the need to consult the third reviewer. Fifty seven percent of the studies received a score of seven or above which suggests the results were less likely to be bias ${ }^{(12,15,17,18,20,22,25)}$. Eight was the highest score obtained: achieved by four studies ${ }^{(12,}$ $15,17,18)$. Three studies obtained a score of less than five (cutoff value) so were excluded from the systematic review due to their high risk of bias ${ }^{(26-28)}$. Most studies obtained points for the method used to conduct the outcome measures and use of appropriate statistical analysis. As is the case with a lot of clinical trials, it was not possible to blind participants to the intervention allocation that they would participate in, therefore no studies received a score for this quality. Common shortcomings in methodological quality were lack of allocator blinding and detail about attrition and intention to treat analysis.

\section{Participant characteristics and sample size}

The selected studies were undertaken across different continents: Europe $(n=8)$, North America $(n=1)$, South America $(n=1)$, Canada $(n=1)$ and Asia $(n=1)$. The important characteristics and significant results of the studies were summarized (Table 2). The total number of participants in the studies was 901 (mean age 82.6 (3.5) years). All participants lived in nursing homes and were recognized as living with a dementia.

The severity of dementia varied across the studies from mild to severe. Some studies only included individuals with a specific level of severity, others included individuals with a range of severity levels; the most common selection was individuals across the spectrum of mild to severe dementia $(n=4)$. When determining the severity, tools such as the Clinical Dementia Rating and the Global Deterioration Scale should be used as they assess multiple aspects of dementia: cognition, emotion, memory and functional ability ${ }^{(29)}$; both scale were used in one study each ${ }^{(20,21)}$. Often, cognitive screening tools such as the Mini Mental State Examination (MMSE) are used to determine severity of dementia, and in this review it was the most commonly used approach to assessing severity of dementia $(n=10)$. This tool is limited because it only assesses one element of dementia and is influenced by the educational level of an individual ${ }^{(29)}$. Due to the variation of tools used and 
the different aspects of dementia assessed, it was not possible to compare the effects of physical exercise on the severity of dementia across the studies.

Information about the functional ability of participants was limited in all studies. Only one included a sub analysis of participants that had low mobility (determined by the inability to walk more than 91.4 meters in the six minute walk test at baseline); they found that the changes were the same as in the whole group analysis though any improvements made were more pronounced in the low mobility group ${ }^{(14,22,23)}$. One study did not mention the functional ability of the participants ${ }^{(18)}$ and all other studies only provided information on the required functional ability as part of the inclusion criteria. The level of functional ability and how it was measured varied widely across the studies. Some studies only stated tasks that participants were able to complete: sit and engage in simple activities ${ }^{(16,20)}$, have no hand disabilities ${ }^{(25)}$, be able to walk with or without assistance (type and level of assistance varied between studies) $)^{(12,13,15,19,24)}$, or be dependent in an activity of daily living $(A D L)$ and have the ability to walk with or without assistance ${ }^{(14,22,23)}$. Other studies used more rigorous methods of functional assessment, such as the Barthel Index $(\mathrm{BI})^{(21)}$, the Performance Orientated Mobility Assessment ${ }^{(21)}$ and the German scale of medical care ${ }^{(17)}$. The heterogeneity of functional ability among participants and how this was assessed across the studies meant it was not possible to determine which level of function benefited the most from a physical exercise intervention.

\section{Control group type}

All studies used a control group. Five control groups received some form of interaction: four had individual social visits/conversations ${ }^{(12-14,16,22,23)}$ and one participated in group reading sessions ${ }^{(25)}$. All groups received the control activity for the same duration and frequency as the intervention group. Six control groups received no extra input but continued to participate in the usual care and activities provided at the nursing homes ${ }^{(15,17}$, 19-21, 24). One control group had no motor intervention (the meaning of 'no motor intervention' was not clarified by the authors) ${ }^{(18)}$.

\section{Intervention characteristics}

Three of the studies had two intervention groups ${ }^{(14,18,22-24)}$. The proportion of individual and group settings were similar: eight group interventions ${ }^{(15-18,20,24,25)}$, six individual interventions ${ }^{(12-14,18,21-23)}$ and it was unclear 
in one study ${ }^{(19)}$. The intervention characteristics varied across the studies (Table 2), though some similarities were found in terms of the type of physical exercise completed: (i) Multimodal ( $n=6)$; (ii) walking ( $n=5)$; (iii) music and movement ( $n=2)$; and (iv) hand exercises $(n=1)$. One study had a second intervention group that did not involve physical exercise, instead it was a conversation only group ${ }^{(24)}$. The multimodal groups included interventions that combined different types of physical exercises targeting strength, balance, flexibility, aerobic capacity, cognition, functional ability and/or coordination ${ }^{(14,15,17-19,22,23)}$. The most common combination was physical exercises in sitting and standing for strength, balance and flexibility combined with walking $^{(14,15,22,23)}$. All the walking groups were supervised and occurred inside, four were individual sessions ${ }^{(12-}$ ${ }^{14,21-23)}$ and one was completed in pairs ${ }^{(24)}$. The music and movement groups focused more on the music and involved generalized movement of the arms and legs ${ }^{(16,20)}$. The hand exercises group only completed hand exercises, although not common, was included as this systematic review which considered all types of physical exercise $^{(25)}$. This was similar to the open inclusion criteria adopted in the Cochrane systematic review completed in $2013^{(5)}$. Older individuals living with a dementia in nursing homes are often frail and not able to participate in physical activities such as walking or standing exercises ${ }^{(30)}$. It is important that different types of physical exercise interventions, such as hand exercises are considered to accommodate varied abilities, and why the hand exercises study was included in this systematic review.

The duration of the intervention varied greatly among the studies from four weeks to 52 weeks (mean duration 17.4 (11.5) weeks). There was also variability in the frequency (mean 4.5 (1.4) sessions per a week) and length (mean 49.3 (30.2) minutes) of the interventions. In most studies, the intensity was not stated $(n=10)$ and of the two studies that did, it was moderate ${ }^{(15,21)}$. The most common parameters were 5 sessions per week $(n=6), 30$ minutes per session $(n=8)$ at moderate intensity $(n=2)$. These parameters were similar to the recommendation by the American College of Sports Medicine for 'apparently healthy adults' which advised 5 or more sessions of moderate intensity physical exercise or 3 or more sessions of high intensity physical exercise for 30 to 60 minutes per week ${ }^{(3)}$.

Compliance of the physical exercise intervention was only detailed in five studies; one only had a mean compliance rate of $33 \%^{(15)}$, whilst the others ranged from 91 to $100 \%^{(12,13,19,21)}$. Four other studies stated that 
compliance was monitored but no numerical data was provided ${ }^{(14,18,20,22,23,25)}$. Three studies did not discuss compliance $^{(16,17,24)}$. Even though compliance was only detailed in less than half of the studies, majority of those that did showed a high compliance from individuals living with a dementia in nursing homes.

\section{Outcome measures}

A range of outcome measures were used $(n=38)$ to evaluate different aspects of health and well being, such as cognitive function $(n=10)$, mood and depression $(n=8)$, functional ability $(n=5)$, mobility $(n=4)$ and unmet needs $^{*}(n=4)$. Other areas included balance, agitation, communication, activity levels and nutrition. Generally, outcome measures were collected at baseline and endpoint with three studies also completing data collection halfway through the intervention and four with a follow up at six or 24 weeks post intervention.

\section{Psychological health and well being outcomes}

\section{Cognition}

Seven studies used outcome measures that evaluated cognition ${ }^{(12,16-19,21,25)}$; the MMSE was the most commonly used $^{(16,18,21,25)}$ and produced significant results in two studies ${ }^{(16,21)}$. In one study the music and movement group's MMSE score improved significantly from 12.9 (5) to $15.5(4.4)$ and at the end of the study the score was 2.7 greater than the control group. This represented a medium effect size of 0.5 ; the multimodal intervention group was clinically relevant ${ }^{(16)}$. The music and movement group also significantly improved in median category fluency score (one of the subscales of the Amsterdam Dementia Screening Test 6) from 10 to 14 , compared to the control group which only increased by $0.5^{(16)}$. In the other study the interaction between groups could not be properly interpreted, however they did find the MMSE score of the control group decreased significantly from 12 (2) to 6 (2) over time, while the walking group only decreased by one point ${ }^{(21)}$.

Other cognition outcome measures used which showed significant changes include: The Nurses' Observation Scale for Geriatric Patients (NOSGER) which found significant improvements in the multimodal group (total score and memory subscale) over time while the control group remained unchanged ${ }^{(17)}$; the French Rapid

\footnotetext{
*Healthcare practitioners and researchers working in the field of dementia care describe 'unmet needs' as those displayed as behaviors by individuals which cannot be easily explained, for example constant calling out, performing repetitive mannerisms and making strange noises.
} 
Evaluation of Cognitive Function test that found the multimodal group significantly improved over time from $26.8(6.4)$ to $30.4(7.7)$ which was significantly greater than the control group's post intervention score of 23.2 (8.4). These findings also demonstrated a significant correlation with the walking parameters (speed, stride length and double limb support time) assessed as part of this study ${ }^{(19)}$; and the Brief Cognitive Screening Battery (BCSB) that showed even though there was a global decline in cognition in the two multimodal groups and the control group, the multimodal group that was led by a multidisciplinary team declined at a significantly slower rate in the Clock Drawing Test and Verbal Fluency Test (components of the BCSB) compared to the control group ${ }^{(18)}$. Other cognitive outcome measures used were the Rivermead Behavioral Memory Test, Eight Words Test, Digit Span, Category and Letter Fluency Tests which were used in two studies but no significant changes were observed ${ }^{(12,25)}$.

\section{Mood and Depression}

Mood and depression categories have been combined as one for the purpose of this systematic review as all studies that stated they assessed the benefits of physical exercise on mood and/or depression looked at both similarly. Mood and depression was evaluated in four studies using a variety of outcome measures ${ }^{(15,17,22,23,}$ 25). One study used the Alzheimer's Mood Scale (AMS), Dementia Mood Assessment Scale (DMAS), Cornell Scale for Depression in Dementia (CSDD) and the Observed Affect Scale ${ }^{(22,23)}$. The study compared the effect of a multimodal physical exercise intervention with walking and a control group on the mood of participants and also completed a sub analysis of those with depression (determined by a score of seven or higher on the CSDD). The multimodal group showed the most improvement, followed by the walking group and then the control group. However, this was only significant for some of the outcome measures when differences between the group's baseline MMSE, physical ability, treatment intensity, depression and affect or mood score were controlled. There were differences in the DMAS posttest adjusted scores for each group, though preplanned contrasts indicated that only the difference between the comprehensive exercise groups score (19.7) and the control group (33.1) was significant. The control group had an adjusted posttest AMS negative subscale score of 64.2 which was significantly poorer than the multimodal group score of 46.9 and the walking group score of 53, the difference between the two physical exercise intervention groups was not significant ${ }^{(23)}$. This was also reflected in the sub analysis of depressed individuals living with a dementia which demonstrated a significant difference in the posttest adjusted score of the negative subscale of the AMS of the control group 
(72.3) when compared to the multimodal group (55.2) and the walking group (52), again the difference between the two physical exercise intervention groups was not significant ${ }^{(22)}$.

Other studies showed mixed results in mood and depression using different outcome measures: In one study the mood subscale of the NOSGER significantly improved over time in the multimodal group by one point (moderate effect size) but remained the same in the control group, however no relative advantage of the change between the groups was found ${ }^{(17)}$; another study used a combination of the Symptoms Check List and the GDS, in the pre protocol analysis a significant reduction in the combined feelings of anxiety and depression in the hand exercise group was found $(t(22)=2.7, p=0.01)$ whereas there was no change in the control group ( $t$ $(23)=-1.7, p=0.1)^{(25)}$; and one study that compared a multimodal physical exercise intervention to a control group found no significant changes with the Montgomery and Asberg Depression Rating Scale ${ }^{(15)}$.

\section{Agitation}

One study evaluated the effect on agitation using the Cohen Mansfield Agitation Inventory (CMAI) and found a significant improvement over time in the music and movement group which was also significantly different to the control group at the halfway and endpoint of the study; halfway difference of $0.6(0.2)$ and endpoint difference of $1.1(0.4)^{(20)}$.

\section{Unmet needs}

Four studies used outcome measures that evaluated unmet needs ${ }^{(15-17,24)}$. One study demonstrated a change in the 'need for help' unmet needs subscale of the Dutch version of the Stockton Geriatric Rating Scale in the control group; a significant improvement from 23 to 17.5 at the intervention halfway point, then a significant reverse trend at the endpoint as the score increase back to 21 . The music and movement group improved, as with the between group difference, it was not significant ${ }^{(16)}$. The study which used the NOSGER did not show any significant changes but was able to show clinical relevance of the multimodal group which had a moderate effect size for the sub scales social behavior $(d=0.54)$ and challenging behavior $(d=0.32)^{(17)}$. The other studies which used the Neuropsychiatric Inventory ${ }^{(15)}$ and the London Psychogeriatric Rating Scale (LPRS) ${ }^{(24)}$ did not show any significant changes in relation to unmet needs. 


\section{Communication}

Communication was considered in one study using the Functional Assessment of Communication Skills for Adults. This study compared a walking and conversation group to a conversation only group and a control group; no statistically significant changes were found ${ }^{(24)}$.

\section{Physical health and well being outcomes}

\section{Mobility}

Five studies evaluated mobility ${ }^{(14,15,19,21,24)}$. One study used the six meter walk test and showed significantly improved efficiency in walking in the multimodal group over time and when compared to the control group, as demonstrated through increased walking speed ${ }^{(15)} ; 0.08 \mathrm{~m} / \mathrm{s}$ after six months which was sustained after 12 months compared to only $0.04 \mathrm{~m} / \mathrm{s}$ after six months and $0.03 \mathrm{~m} / \mathrm{s}$ after 12 months for the control group ${ }^{(15)}$. The six minute walk test showed mixed results in the two studies that adopted it. One study had two intervention groups, multimodal and walking and a control group which all showed improvement though the changes were not significant ${ }^{(14)}$. The other assessed the effect of walking against a control group and found the walking group distance significantly improved from 245 (31) meters (m) to 294 (49) m, while the control group significantly reduced from 238 (47) m to 168 (34) m; a significant difference of $134 \mathrm{~m}^{(21)}$. Another study which considered the effect of walking and conversation used the two minute walk test but no significant changes were found ${ }^{(24)}$. The Locometer was used in another study and again provided results in favor of the multimodal group which improved in all the parameters assessed (speed, stride length and double limb support) while the control group decreased ${ }^{(19)}$. The double limb support time changes were significant within and between both groups; $0.2(0.04)$ seconds $(\mathrm{sec})$ to $0.1(0.03) \mathrm{sec}$ in the multimodal group compared to a change of $0.13(0.04)$ sec to $0.14(0.04)$ sec for the control group ${ }^{(19)}$. These changes demonstrated a significant improvement in the efficiency of mobility and balance of the individuals in the multimodal group.

\section{Balance}

Balance was evaluated in two studies ${ }^{(15,18)}$, both used the Get Up and Go Test, though they were assessed in different ways; one used a score system ${ }^{(15)}$ and the other used time ${ }^{(18)}$, neither produced significant results and neither did the One Leg Balance Test ${ }^{(15)}$. The Berg Balance Scale was the only measure that showed significant changes over time in the two multimodal groups (multidisciplinary approach and physical therapy only) and 
control group; the multidisciplinary group showed the most significant improved score from 39.5 (1.9) to 41.7 (2.4), the physical therapy only group also improved from 37.4 (2) to 37.7 (2.8), while the control group decreased from 35.2 (3) to $27.4(3)^{(18)}$. There was no significant difference in the between group analysis ${ }^{(18)}$.

\section{Functional ability}

Five studies used outcome measures that considered ability to complete ADLs and amount of care time required $^{(14,15,17,21,24)}$. The most commonly used measure was the $\mathrm{BI}^{(17,21)}$. Both studies showed that the $\mathrm{BI}$ improved in the intervention groups (multimodal and walking) though it was only significant in the walking study, where there was an improvement in the score from 34 (4) to 42 (4) which was significantly better than the control group which decreased from $35(6)$ to $32(6)^{(21)}$. Another study showed significant improvement in ability to transfer from one surface to another in the multimodal group using the Acute Care Index Function measure while the walking group and control group both declined ${ }^{(14)}$. In another study that compared a multimodal group to a control group, the Katz Index of ADLs score significantly reduced for both groups which demonstrated deterioration in functional ability. However, the rate of decline was significantly slower in the multimodal group (declined from $3.2(1.3)$ to $2.6(1.5)$ ) compared to the control group (changed from 3.1

(1.3) to $2.2(1.5))^{(15)}$. There were no significant findings in the study that used the LPRS to assess physical disability $^{(24)}$ or the study that assessed care time required using the Resource Utilization in Dementia- Formal Care tool ${ }^{(17)}$.

\section{Activity level}

Two studies evaluated the effect of physical exercise intervention on individual's activity level, including daily activity patterns of rest activity patterns and sleep disturbance, using an Actiwatch ; there were no significant findings in either study ${ }^{(13,25)}$.

\section{Nutrition}

One study considered nutrition using the Mini Nutritional Assessment (MNA) and no change was found in either group ${ }^{(15)}$. 


\section{Discussion}

The results of this systematic review showed there was emerging evidence that physical exercise has a positive effect on health and well being of individuals living with a dementia in nursing homes. Nine of the 12 studies reviewed showed either significant improvement in the intervention group and/or deterioration in the control group in at least one health and well being outcome measure. Cognition, mood and depression, agitation, unmet needs, mobility, balance and functional ability all showed significant improvements. Mood and depression and agitation showed the most consistent improvement as $75 \%$ or more of the studies that used these types of outcome measures had a positive effect in at least one outcome measure; three studies showed improvement in mood and depression ${ }^{(17,22,23,25)}$ and the one study that assessed agitation showed an improvement ${ }^{(20)}$. The results from these studies appear trustworthy and have a low risk of bias as they were high quality studies; the majority scored a 7 or higher in the methodological assessment. It could be hypothesized that both these categories of outcome measures showed the most consistent improvement as the physical exercise interventions engaged the participants and encouraged interaction with others which may have given them a feeling of belonging and purpose whilst distracting them from negative feelings. This could have also been the case in the study which demonstrated clinical relevance in some of the sub categories of the NOSGER (social behavior and challenging behavior) which was used to assess unmet needs ${ }^{(17)}$. However, the other three studies that considered unmet needs did not find significant changes. In one study treatment was only provided twice a week which may not have been enough to significantly affect this outcome and a more targeted approach to unmet needs may be necessary ${ }^{(15)}$. The results of the other studies were limited by a small sample size ${ }^{(16,24)}$ and low methodological quality (lack of blinding and detail about the intervention and attrition) $)^{(16)}$.

Cognition was another area that showed improvement with physical exercise and there are several hypotheses for this, including improved blood circulation in the brain and stimulation of synaptic and/or neuronal function ${ }^{(19,21)}$. Of the five studies that found significant changes in cognition, three used a multimodal physical exercise intervention ${ }^{(17-19)}$ and one was a music and movement group ${ }^{(16)}$. Whereas the two that showed no significant changes in cognition involved either walking ${ }^{(12)}$ or hand exercises ${ }^{(25)}$. This may suggest that physical exercise interventions that involve multiple tasks and are aerobic are the most effective in producing cognitive changes for individuals living with a dementia in nursing homes. A previous study in older healthy individuals 
showed aerobic activity improved cognition but anaerobic activity did not ${ }^{(31)}$. Even though walking is considered an aerobic activity the study that found no significant changes may not have been intense or long enough to effect cognition, as participants walked at a self-selected speed and were encouraged to rest as required over a six week period ${ }^{(12)}$. This was different to the other walking study that found significant changes which lasted 24 weeks and had participants walk at a moderate intensity ${ }^{(21)}$. The study that did not show any changes in cognition also had a large proportion of individuals with cardiovascular disease (89\%) which leads to reduced cardiac output and may reduce cerebral perfusion, limiting the effects of aerobic exercise $^{(12)}$. The results from the studies that assessed cognition should be considered with caution as even though four were high quality studies ${ }^{(12,17,18,25)}$, three were of lower quality ${ }^{(16,19,21)}$ so there is a risk that bias was introduced which along with the common limitations of a small sample size and short duration could have influenced the results. Caution should also be taken when interpreting results from cognition outcome measures as most requires a minimum level of comprehension and schooling. They are also influenced by depression which is highly prevalent in older individuals living with a dementia ${ }^{(18)}$. Both these factors can alter the results obtained from outcome measures such as the MMSE (most commonly used cognition outcome measure in this systematic review) and the Clock Drawing Test.

Along with the psychological benefits some studies were able to demonstrate physical benefits for individuals living with a dementia in nursing homes. Seven studies used various outcome measures that assessed the effect of a physical exercise intervention on mobility, balance and/or functional ability ${ }^{(14,15,17-19,21,24)}$, six found changes in favor of the intervention group which were significant in all but one study ${ }^{(17)}$. This study used the BI to assess functional ability which may have not been sensitive enough for the study population that included individuals with mild to severe dementia. The BI has very coarse categories when rating independence which are less reliable when assessing individuals with a cognitive impairment ${ }^{(32)}$. All studies that assessed mobility, balance and/or functional ability used a multimodal and/or walking intervention. It could be hypothesized that these type of physical exercise were beneficial as they targeted and included elements of the outcome measures, such as walking, strength, flexibility and range of movement. All of the studies lasted at least 15 weeks which provided sufficient time for changes in physical function to occur. The literature suggested participation in physical exercise for a minimum of 12 weeks is required for physiological adaptations to occur in frail, older individuals ${ }^{(33)}$. However, not all studies showed significant changes in physical outcome 
measures. Two studies showed there were no positive influence on activity levels with the use of an Actiwatch $^{(12,13)}$. Both studies were by the same authors and considered the effect of a walking intervention (same parameters) on individuals living with a mild to moderately severe dementia, one focused on nighttime restlessness ${ }^{(13)}$ and the other cognition ${ }^{(12)}$. Both papers were checked by three reviewers to ensure they were not from the same study. A third study that considered the effect of walking and conversation on functional ability, using the LPRS, did not find significant changes either ${ }^{(24)}$. As all studies had similar parameters and findings it could be hypothesized that walking or the parameters set were not effective in targeting the outcomes measures used (the duration was only six weeks in two studies and the intensity was not stated but appeared low in all studies) or were not specific enough to produce changes for individuals living with a dementia. A previous study did find a multimodal intervention only had a positive effect on sleep disturbances of individuals living with a severe dementia in nursing homes ${ }^{(34)}$. Another study did not find any benefit of a multimodal physical exercise intervention on nutrition as assessed by the $\mathrm{MNA}^{(15)}$. This may have been because the MNA was not sensitive enough for this study population, or a more targeted approach to nutrition was required. In terms of methodological quality there was a mixture of high and low quality studies $(50 \%$ each) that assessed physical outcome measures so the overall results should be considered with caution.

A high proportion of studies used a multimodal intervention, including different types of physical exercises, functional tasks and cognitive tasks. These studies showed the most benefit as all five studies had a significant improvement in at least one health and well being outcome in the intervention group(s) compared to the control group ${ }^{(14,15,17-19,22,23)}$. Strength and balance were the most common components in all the studies that included a multimodal group. In one studies that had a multimodal group, walking only group and control group, the multimodal group showed the most improvement; mobility and functional ability outcome measures were significantly better than the other two groups involved, while improvements in the mood and depression outcome measures were only significant when compared to the control group only ${ }^{(14,22,23)}$. The studies that investigated walking interventions did have some significant findings, though they did not appear to be as beneficial as a multimodal approach. Five studies considered the effect of walking on individuals with a dementia ${ }^{(12-14,21-24)}$ but only two of these found significant improvement in health and well being ${ }^{(21,22)}$. The other two studies either had inconclusive results or found that there was no benefit. This may have been because the walking activity was not intense or long enough to produce any significant changes. The studies 
that used music and movement found significant improvements ${ }^{(16,20)}$, as did the study that used hand exercises $^{(25)}$. These studies and those in the multimodal group used activities that were not part of the usual day to day routine, while walking was an automatic task completed daily by the participants. This could explain why these types of physical exercise showed more significant improvements than walking alone. They also involved more social interaction and touch which could have enhanced the results ${ }^{(23)}$ and involved activities that targeted the various elements that are required to improve the outcome measures. Examples include strengthening and balance exercise to help improve mobility and aerobic exercises to stimulate cerebral blood circulation and function in cognition.

Physical exercise interventions that are different to an individual's usual routine and targets more than one aspect could be the most beneficial for individuals living with a dementia in nursing homes. Physical Therapists would be the most appropriate to complete such interventions due to their knowledge and understanding of physical exercise. Three of the studies conducted physical exercise interventions that were completed or supervised by a Physical Therapist or Physical Therapy student and all showed significant improvements in psychological and physical health and well being outcome measures ${ }^{(14,18,21-23)}$. This demonstrates the importance of and need to increase the role of Physical Therapists in dementia care in the nursing home setting, as currently their skills and knowledge are an untapped resource in this area. It would also be important to consider the economic benefits of providing physical exercise programs by Physical Therapists in nursing homes.

The most common limitation found in the review was a small sample size; highlighted as a limitation in $50 \%$ of the studies. This is most likely due to reduced feasibility, ethical concerns with consent and high attrition rate in this population group. In some studies the duration of the intervention period was too short which limited the possible effect of the intervention. There were four studies that went for six weeks or less: two found no benefit of physical exercise for individuals living with a dementia in nursing homes ${ }^{(12,13)}$. Another issue identified was the lack of detail, specifically of the method and the intervention used. This reduced the reliability and validity of some studies, introducing bias and reducing the ability to replicate all or elements of the studies. Further, high quality research, for longer periods and with larger sample sizes is required to help support the current evidence. There is also a need to determine the optimum parameters of physical exercise 
interventions, such as type, frequency, length and intensity. This evidence will help practitioners plan and implement the most effective physical exercise program for individuals living with a dementia in nursing home.

This systematic review considered all relevant studies identified from a search across several databases and from snowballing. It differed from the Cochrane review ${ }^{(5)}$ completed in 2013 as this systematic review focused on studies set in nursing homes only and, unlike the Cochrane review, did not limit the type of outcomes considered and including more than simply the effects of physical exercise on cognition, ADLs, challenging behavior, depression and mortality. Due to these differences this systematic review considered three studies not included in the Cochrane systematic review. It provided further evidence and supported the findings of the Cochrane systematic review, on the beneficial effects of physical exercise interventions for individuals living with a dementia in nursing homes. Other limitations were that studies could have been missed due to an inability to access other databases and non-English publications. This review only considered RCTs and cluster RCTs, other types of studies that were excluded could have provided further relevant evidence on this topic. Due to the high variability between the studies in terms of the type of physical exercise, parameters used and outcome measures assessed it was not possible to complete a meta analysis. It was also not possible to evaluate which levels of functional ability and severity of dementia benefited the most from physical exercise due to the wide variation in tools/methods used to assess these aspects and the lack of detail in many of the studies.

\section{Conclusion}

The findings of this systematic review suggested that physical exercise positively effects health and well being of individuals living with a dementia in nursing homes, particularly when a multimodal approach that involves a combination of activities was utilized. Interventions that combined strength, balance, flexibility and endurance (most often in the form of walking) were the most common combination to produce significant improvements in the health and well being of individuals living with a dementia in nursing homes. Not only over time but also when compared to other interventions, such as walking and seated social activities. A number of studies demonstrated significant improvements in areas such as cognition, agitation, mood, mobility and functional ability. The duration, length and frequency set in the studies reviewed varied greatly and even though the optimum parameters of physical exercise interventions have not yet been determined, 
this systematic review has shown that physical exercise for at least 30 minutes twice a week can produce significant improvements. No conclusions in regards to the intensity of the physical exercise interventions or severity of dementia could be drawn from this systematic review as this information varied in the few studies that did documented it.

This is an emerging area of research that has gained momentum over recent years and as the population ages the demand on healthcare will increase so it is important that effective dementia care is in place. To ensure this can happen, further high quality research for longer periods and with larger sample sizes are warranted to build on current evidence. This will help to determine the optimum parameters of physical exercise interventions for individuals living with a dementia in nursing homes and address other key issues, such as falls, sleep, social interaction and self esteem. This will help to guide practitioners who work in nursing homes and utilize the skills of health professionals such of Physical Therapists, so that a rehabilitation approach to dementia care can be adopted, optimizing the quality of life of individuals living with a dementia in nursing homes. 


\section{Acknowledgements}

All authors provided concept/idea/research design. The authors acknowledge the funding received from the University of Wollongong PhD Scholarship and the IHMRI Dementia Summer Scholarship. The authors declare there was no conflict of interest. 
1. World Health Organisation. Dementia Fact Sheet Number 3622012 [24/05/2014]. Fact Sheet number 362]. Available from: www.who.int/mediacentre/factsheets/fs362/en/.

2. Seitz DP, Brisbin S, Herrmann N, Rapoport MJ, Wilson K, Gill SS, et al. Efficacy and Feasibility of Nonpharmacological Interventions for Neuropsychiatric Symptoms of Dementia in Long Term Care: A Systematic Review. Journal of the American Medical Directors Association. 2012;13(6):503-+. 3. Garber CE, Blissmer B, Deschenes MR, Franklin BA, Lamonte MJ, Lee I-M, et al. Quantity and Quality of Exercise for Developing and Maintaining Cardiorespiratory, Musculoskeletal, and Neuromotor Fitness in Apparently Healthy Adults: Guidance for Prescribing Exercise. Medicine \& Science in Sports \& Exercise. 2011;43(7):1334-59 10.249/MSS.0b013e318213fefb.

4. Pitkälä K, Savikko N, Poysti M, Strandberg T, Laakkonen M-L. Efficacy of physical exercise intervention on mobility and physical functioning in older people with dementia: A systematic review. Experimental Gerontology. 2013;48(1):85-93.

5. Forbes D, Thiessen EJ, Blake CM, Forbes SC, Forbes S. Exercise programes for people with dementia. Cochrane Database Syst Rev. 2013;12.

6. Australian Institute of Health and Welfare. Dementia in Australia. In: Welfare AloHa, editor. Canberra: AlHW; 2012.

7. Australian Government. Public Health Act. 2010.

8. Australian Bureau of Statisitics. People identified as havig dementia or ALzheimer's disease 2012 [updated 09/04/201324/05/14]. 4102.0:[Available from: http://www.abs.gov.au/AUSSTATS/abs@.nsf/Lookup/4102.0Main+Features50Dec+2012.

9. Aman E, Thomas DR. Supervised Exercise to Reduce Agitation in Severely Cognitively Impaired Persons. Journal of the American Medical Directors Association. 2009;10(4):271-6.

10. Moher D, Liberati A, Tetzlaff J, Altman DG. Preferred Reporting Items for Systematic Reviews and Meta-Analyses: The PRISMA Statement. Ann Intern Med. 2009;151(4):264-9.

11. The Joanna Briggs Institute. Joanna Briggs Instititute Reviewers' Manual: 2014 edition. Adelaide: Joanna Briggs Institute; 2014.

12. Eggermont LH, Swaab DF, Hol EM, Scherder EJ. Walking the line: a randomised trial on the effects of a short term walking programme on cognition in dementia. Journal of neurology, neurosurgery, and psychiatry. 2009;80(7):802-4.

13. Eggermont LHP, Blankevoort CG, Scherder EJA. Walking and night-time restlessness in mildto-moderate dementia: a randomized controlled trial. Age And Ageing. 2010;39(6):746-9.

14. Roach KE, Tappen RM, Kirk-Sanchez N, Williams CL, Loewenstein D. A Randomized Controlled Trial of an Activity Specific Exercise Program for Individuals With Alzheimer Disease in Long-term Care Settings. J Geriatr Phys Ther. 2011;34(2):50-6.

15. Rolland Y, Pillard F, Klapouszczak A, Reynish E, Thomas D, Andrieu S, et al. Exercise Program for Nursing Home Residents with Alzheimer's Disease: A 1-Year Randomized, Controlled Trial. Journal of the American Geriatrics Society. 2007;55(2):158-65.

16. van de Winckel A, Feys $H$, De Weerdt W, Dom R. Cognitive and behavioural effects of musicbased exercises in patients with dementia. Clinical Rehabilitation. 2004;18(3):253-60.

17. Luttenberger K, Donath C, Uter W, Graessel E. Effects of multimodal nondrug therapy on dementia symptoms and need for care in nursing home residents with degenerative dementia: a randomized-controlled study with 6-month follow-up. J Am Geriatr Soc. 2012;60(5):830-40.

18. Christofoletti G, Oliani MM, Gobbi S, Stella F, Gobbi LTB, Canineu PR. A controlled trial on the effects of motor intervention on balance and cognition in institutionalized elderly patients with dementia. Clinical Rehabilitation. 2008;22(1):618-26.

19. Kemoun G, Thibaud M, Roumagne N, Carette P, Albinet C, Toussaint L, et al. Effects of a Physical Training Programe on Cognitive Function and Walking Effciency in Elderly Persons with Dementia. Dementia and Geriatric Cognitive Disorders. 2010;29:109-14. 
20. Sung H-c, Chang S-m, Lee $\mathrm{W}-\mathrm{I}$, Lee M-s. The effects of group music with movement intervention on agitated behaviours of institutionalized elders with dementia in Taiwan. Complementary Therapies in Medicine. 2006;14(2):113-9.

21. Venturelli $M$, Scarsini $R$, Schena $F$. Six-month walking program changes cognitive and ADL performance in patients with Alzheimer. Am J Alzheimers Dis Other Dement. 2011;26(5):381-8.

22. Williams $\mathrm{CL}$, Tappen RM. Exercise training for depressed older adults with Alzheimer's disease. Aging \& Mental Health. 2008;12(1):72-80.

23. Williams CL, Tappen RM. Effect of Exercise on Mood in Nursing Home Residents With Alzheimer's Disease. American Journal of Alzheimer's Disease and Other Dementias. 2007;22(5):38997.

24. Cott CA, Dawson P, Sidani S, Wells D. The effects of a walking/talking program on communication, ambulation, and functional status in residents with Alzheimer disease. Alzheimer disease and associated disorders. 2002;16(2):81-7.

25. Eggermont LHP, Knol DL, Hol EM, Swaab DF, Scherder EJA. Hand motor activity, cognition, mood, and the rest-activity rhythm in dementia: A clustered RCT. Behavioural Brain Research. 2009;196(2):271-8.

26. Stevens J, Killeen M. A Randomised Controlled Trial Testing the Impact of Exercise on Cognitive Symptoms and Disability of Residents with Dementia. Contemporary Nurse. 2006;21(1):3240.

27. Venturelli M, Magalini A, Scarsini R, Schena F. From Alzheimer's Disease Retrogenesis: A New Care Strategy for Patients With Advanced Dementia. Am J Alzheimers Dis Other Dement. 2012;27(7):483-9.

28. Francese T, Sorrell J, Butler FR. The effects of regular exercise on muscle strength and functional abilities of late stage Alzheimer's residents. American Journal of Alzheimer's Disease and Other Dementias. 1997;12(3):122-7.

29. Choi SH. Interchanging Scores Between Clinical Dementia Rating Scale and Global Deterioration Scale. Alzheimer disease and associated disorders. 2003;17(2):98-105.

30. Ang YH, Au SYL, Yap LKP, Ee CH. Functional decline of the elderly in a nursing home. Singapore Medical Journal. 2006;47(3):219-24.

31. Kramer AF, Hahn S, Cohen NJ, Banich MT, McAuley E, Harrison CR, et al. Ageing, fitness and neurocognitive function. Nature. 1999;400(6743):418-9.

32. Sainsbury A, Seebass G, Bansal A, Young JB. Reliability of the Barthel Index when used with older people. Age and Ageing. 2005;34(3):228-32.

33. Bonner S, Cousins A. Exercise and Alzheimer's Disease: benefits and barriers. Activities, Adaptation \& Aging. 1996;20(4):21-34.

34. Alessi CA, Yoon EJ, Schnelle JF, Al-Samarrai NR, Cruise PA. A randomized trial of a combined physical activity and environmental intervention in nursing home residents: Do sleep and agitation improve? Journal of the American Geriatrics Society. 1999;47(7):784-91. 
Table 1

Methodological Quality Assessment

\begin{tabular}{|c|c|c|c|c|c|c|c|c|c|c|c|}
\hline \multirow[t]{2}{*}{ Article } & \multicolumn{11}{|c|}{ Methodological Questions* } \\
\hline & 1 & 2 & 3 & 4 & 5 & 6 & 7 & 8 & 9 & 10 & $\operatorname{Total}^{\dagger}(/ 10)$ \\
\hline Christofoletti et al, $2008^{18}$ & Yes & No & Yes & No & Yes & Yes & Yes & Yes & Yes & Yes & 8 \\
\hline Eggermont et al, $2009^{12}$ & Yes & No & No & Yes & Yes & Yes & Yes & Yes & Yes & Yes & 8 \\
\hline Luttenberger et al, $2012^{17}$ & Yes & No & No & Yes & Yes & Yes & Yes & Yes & Yes & Yes & 8 \\
\hline Rolland et al, $2007^{15}$ & Yes & No & Yes & Yes & Yes & No & Yes & Yes & Yes & Yes & 8 \\
\hline Eggermont et al, $2009^{25}$ & No & No & No & Yes & Yes & Yes & Yes & Yes & Yes & Yes & 7 \\
\hline Sung et al, $2006^{20}$ & Yes & No & Yes & No & No & Yes & Yes & Yes & Yes & Yes & 7 \\
\hline Williams and Tappen, $2008^{22, \ddagger}$ & Yes & No & Yes & No & Yes & No & Yes & Yes & Yes & Yes & 7 \\
\hline Cott et al, $2002^{24}$ & Yes & No & No & No & No & Yes & Yes & Yes & Yes & Yes & 6 \\
\hline Venturelli et al, $2011^{21}$ & Yes & No & No & No & Yes & Yes & Yes & Yes & No & Yes & 6 \\
\hline Williams and Tappen, $2007^{23, \ddagger}$ & No & No & Yes & No & Yes & Yes & No & Yes & Yes & Yes & 6 \\
\hline Eggermont et al, $2010^{13}$ & No & No & No & No & No & Yes & Yes & Yes & Yes & Yes & 5 \\
\hline Kemoun et al, $2010^{19}$ & Yes & No & No & No & No & Yes & Yes & Yes & No & Yes & 5 \\
\hline Roach et al, $2011^{14, \ddagger}$ & No & No & No & No & Yes & Yes & Yes & Yes & No & Yes & 5 \\
\hline van De Winckel et al, $2004^{16}$ & Yes & No & No & No & No & Yes & No & Yes & Yes & Yes & 5 \\
\hline Stevens and Killeen, $2006^{26, \S}$ & Yes & No & No & No & No & Yes & No & Yes & Yes & No & 4 \\
\hline Francese et al, $1997^{28, \$}$ & No & No & No & No & No & No & No & Yes & No & Yes & 2 \\
\hline Venturelli et al, $2012^{27, \S}$ & No & No & No & No & No & No & No & Yes & No & Yes & 2 \\
\hline Mean Total Score & 6 & & & & & & & & & & \\
\hline
\end{tabular}

Method: Joanna Briggs Institute Meta-Analysis of Statistics Assessment and Review Instrument critical appraisal tool for randomized/pseudo-randomized control trials.

${ }^{*}$ Methodological questions:

(1) Was the assignment to treatment groups truly random?

(2) Were participants blinded to treatment allocation?

(3) Was allocation to treatment groups concealed from the allocator?

(4) Were the outcomes of people who withdrew described and included in the analysis?

(5) Were those assessing outcomes blind to the treatment allocation?

(6) Were the control and treatment groups comparable at entry?

(7) Were groups treated identically other than for the named intervention?

(8) Were outcomes measured in the same way for all groups?

(9) Were outcomes measured in a reliable way?

(10) Was appropriate statistical analysis used?

${ }^{\dagger}$ Higher score $=$ higher methodological quality.

'Different publication from the same study.

${ }^{5}$ Studies excluded because of methodological score below cut-off point (mean score minus 1 standard deviation). 
Table 2

Physical Exercise for Individuals With Dementia in Nursing Homes-Current Research Summary Table

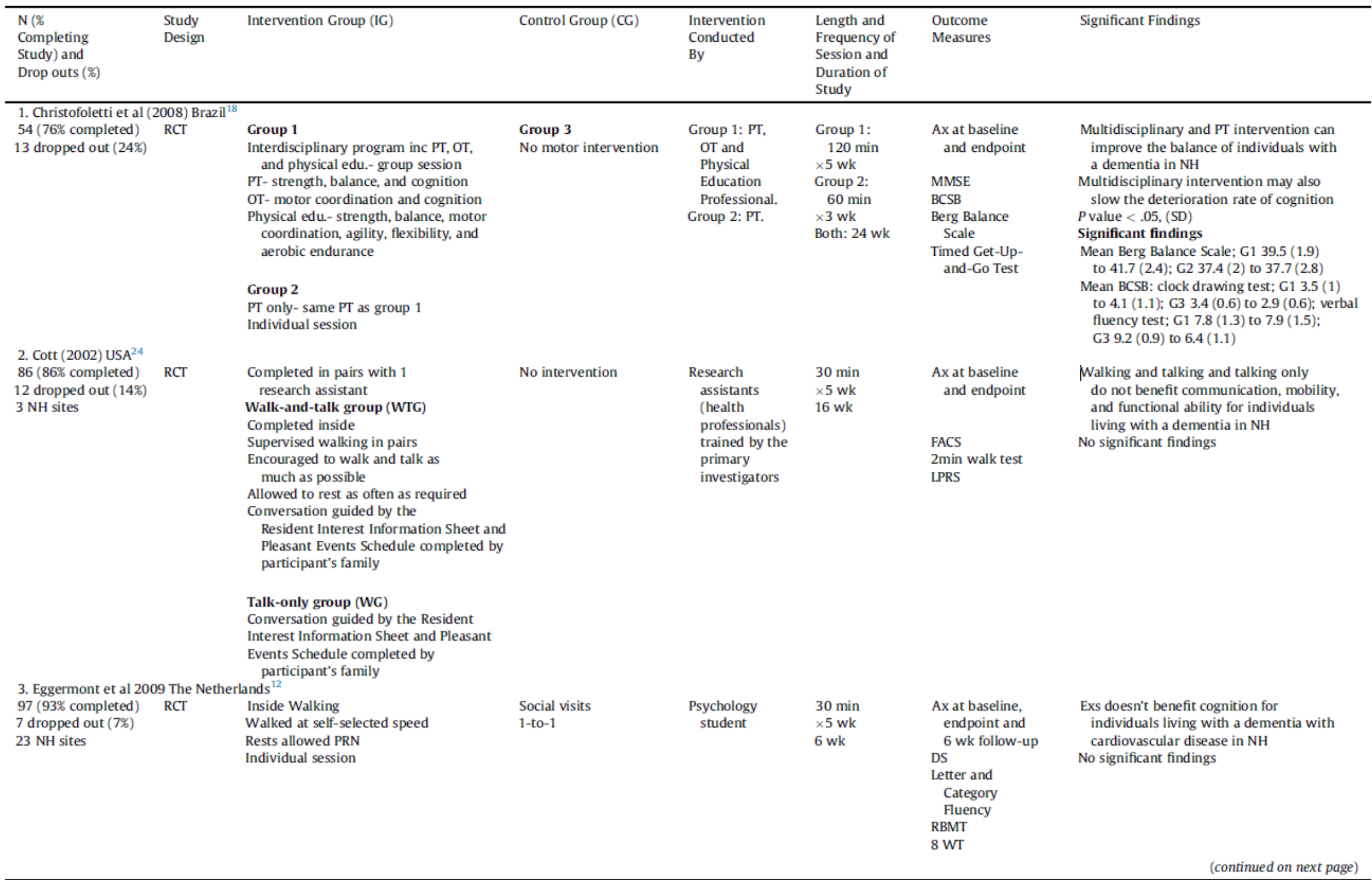


Table 2 (continued)

\begin{tabular}{|c|c|c|c|c|c|c|c|}
\hline $\begin{array}{l}\mathrm{N}(\% \\
\text { Completing } \\
\text { Study) and } \\
\text { Drop outs }(\%)\end{array}$ & $\begin{array}{l}\text { Study } \\
\text { Design }\end{array}$ & Intervention Group (IG) & Control Group (CG) & $\begin{array}{l}\text { Intervention } \\
\text { Conducted } \\
\text { By }\end{array}$ & $\begin{array}{l}\text { Length and } \\
\text { Frequency of } \\
\text { Session and } \\
\text { Duration of } \\
\text { Study }\end{array}$ & $\begin{array}{l}\text { Outcome } \\
\text { Measures }\end{array}$ & Significant Findings \\
\hline \multicolumn{8}{|c|}{ 4. Eggermont et al (2009) The Netherlands ${ }^{25}$} \\
\hline \multirow{12}{*}{$\begin{array}{l}66 \text { (92\% completed) } \\
5 \text { dropped out ( } 8 \%)\end{array}$} & Cluster RCT & Hand movement program & Read aloud group & Recreational & $30 \mathrm{~min}$ & Ax at baseline & Increased attendance to hand motor \\
\hline & & Exs based on movements especially & program & Therapist and & $\times 5 \mathrm{wk}$ & (T1), endpoint & activity program improved mood \\
\hline & & designed for this population & Stories from books & Psychology & $6 \mathrm{wk}$ & (T2) and $6 \mathrm{wk}$ & $P$ value .05 , (SD) \\
\hline & & Morning activity & recommended for this & students & & follow-up (T3) & Significant find ings \\
\hline & & Group session & $\begin{array}{l}\text { population read aloud } \\
\text { by group leader }\end{array}$ & & & RBMT & $\begin{array}{l}\text { Mean GDS; IG } 7.9(6.1) \text { to } 5.9(4.6) \text { to } \\
6.1(4.9)\end{array}$ \\
\hline & & & Followed by conversation & & & $8 W T$ & Mean SCL Anxiety; IG 16.0 (5.1) to \\
\hline & & & based on preselected & & & DS & $13.3(3.3)$ to $12.8(38.5)$ \\
\hline & & & topics from meeting & & & Category Fluency & IG vs CG time $\times$ group interaction; \\
\hline & & & & & & MMSE & mood domain 5.5 \\
\hline & & & & & & & IG vs CG contrast T1-T2; mood domain 3.2 \\
\hline & & & & & & SCL. Anxiety & \\
\hline \multirow{2}{*}{\multicolumn{8}{|c|}{ 5. Eggermont et al (2010) The Netherlands ${ }^{13}$}} \\
\hline & & & & & & & \\
\hline $\begin{array}{l}79 \text { (91\% completed) } \\
7 \text { dropped out }(9 \%)\end{array}$ & RCT & $\begin{array}{l}\text { Walking with a student } \\
\text { Self-selected speed }\end{array}$ & $\begin{array}{l}\text { Social visits from } \\
\text { a student }\end{array}$ & $\begin{array}{l}\text { A student } \\
\text { (type of }\end{array}$ & $\begin{array}{l}30 \mathrm{~min} \\
\times 5 \mathrm{wk}\end{array}$ & $\begin{array}{l}\text { Ax at baseline, } \\
\text { endpoint and }\end{array}$ & $\begin{array}{l}\text { No beneficial effect of walking on } \\
\text { night-time restlessness or other }\end{array}$ \\
\hline $19 \mathrm{NH}$ sites & & Rest PRN & Time of day variable & student not & & 6-wk follow-up & actigraphy parameters \\
\hline & & $\begin{array}{l}\text { Time of day variable } \\
\text { Individual session }\end{array}$ & & stated) & & Actiwatch & No significant find ings \\
\hline \multicolumn{8}{|c|}{ 6. Kemoun et al (2010) France ${ }^{19}$} \\
\hline \multirow{11}{*}{$\begin{array}{l}38 \text { (89\% completed) } \\
7 \text { dropped out }(11 \%)\end{array}$} & RCT & $\times 1$ wk- walking and motor route exs & Usual activities & Not stated & $60 \mathrm{~min}$ & Ax at baseline & Walking program had positive influence \\
\hline & & $\begin{array}{l}\times 1 \text { wk- stamina exs } \\
\times 1 \text { wk- combined stamina, equilibrium } \\
\text { and walking }\end{array}$ & provided at $\mathrm{NH}$ & & $\begin{array}{l}\times 3 \mathrm{wk} \\
15 \mathrm{wk}\end{array}$ & and endpoint & $\begin{array}{l}\text { on spatiotemporal variables concerning } \\
\text { walking and cognitive capabilities } \\
\text { Significant correlation between changes }\end{array}$ \\
\hline & & Each session- warm up for $10 \mathrm{~min}$, & & & & & in ERFC and walking variables \\
\hline & & followed by $40 \mathrm{~min}$ of one of the & & & & ERFC & $P$ value $<.01,(\mathrm{SD})$ \\
\hline & & above activities, then $10 \mathrm{~min}$ & & & & Bessou Locometer & Significant findings \\
\hline & & $\begin{array}{l}\text { relaxation } \\
\text { Unclear if individual or group session }\end{array}$ & & & & and SATEL software & $\begin{array}{l}\text { Mean ERFC; IG } 26.8(6.4) \text { to } 30.4(7.7) \\
\text { CG } 28.3(7.1) \text { to } 23.2(8.4)\end{array}$ \\
\hline & & & & & & & $\begin{array}{l}\text { Mean Walking speed: IG } 0.7(0.1) \text { to } 1.0 \\
(0.2) ; \text { CG } 0.9(0.2) \text { to } 0.8(0.2)\end{array}$ \\
\hline & & & & & & & Mean Stride length; IG $0.9(0.2)$ \\
\hline & & & & & & & to $1.0(0.2) ; C G 1.0(0.20)$ to $0.9(0.2)$ \\
\hline & & & & & & & Mean Double limb support; IG $0.2(0.04)$ \\
\hline & & & & & & & to $0.1(0.03) ; C G 0.13(0.04)$ to $0.14(0.04)$ \\
\hline \multicolumn{8}{|c|}{ 7. Luttenberger et al (2012) Germany ${ }^{17}$} \\
\hline \multirow{7}{*}{$\begin{array}{l}141 \text { ( } 84 \% \text { completed) } \\
22 \text { dropped out ( } 16 \%)\end{array}$} & $\mathrm{RCT}$ & Multicomponent group therapy & Usual activities & 2 ther apists and & $120 \mathrm{~min}$ & Ax at baseline & MAKS significantly and dinically more \\
\hline & & $\begin{array}{l}10 \text { min "spiritual" intro, } 30 \text { min } \\
\text { motor activity, } 10 \text { min break, } \\
30 \text { min individualized cognitive }\end{array}$ & provided at $\mathrm{NH}$ & $\begin{array}{l}1 \text { aide (type of } \\
\text { therapists and } \\
\text { aide not stated) }\end{array}$ & $\begin{array}{l}\times 6 \mathrm{wk} \\
24 \mathrm{wk}\end{array}$ & and endpoint & $\begin{array}{l}\text { effective than usual care for dementia, } \\
\text { especially social unmet needs and ADL. } \\
\text { completion }\end{array}$ \\
\hline & & $\begin{array}{l}30 \text { min individualized cognitive } \\
\text { tasks, } 40 \text { min ADLs }\end{array}$ & & & & BI & $P$ value $<.05,(95 \% \mathrm{Cl})$ \\
\hline & & Groups of 10 & & & & NOSGER & Significant findings \\
\hline & & & & & & RUD-FOCA & $\begin{array}{l}\text { NOSGER mean difference after } 24 \text { wks; } \\
\text { IG sum } 5.3(2.2-8.3) ; \text { IG subscale mood }\end{array}$ \\
\hline & & & & & & & $\begin{array}{l}1.0(0.3-1.7) ; \text { IG subscale memory } \\
1.5(0.6-2.3)\end{array}$ \\
\hline & & & & & & & $\begin{array}{l}\text { NOSGER clinical relevance; moderate } \\
\text { effect size in sub scales social behavior } \\
(\mathrm{d}=0.54) \text { and challenging behavior } \\
(\mathrm{d}=0.32)\end{array}$ \\
\hline
\end{tabular}


8. Rolland et al (2007) France $^{15}$

134 (82\% completed) RCT

24 dropped out ( $18 \%$

$5 \mathrm{NH}$ sites

9. Sung et al (2006) Taiwan

40 (90\% completed)

RCT

Co

individualized Inc walking

(aerobic), strength, balance,

and flexibility training

Interspersed with strength,

flexibility and balance

stations along the trail

Held in the afternoon

Music accompanied sessions

Groups of 2-7

Music with movement interventio

Familiar music that had pleasant,

moderate rhythm, and tempo

involved movements

Conducted in the afternoon

Group session

10. van De Winckel et al (2004) The Netherlands ${ }^{16}$

25 (96\% completed) RCT Music-based seated dance sessions

1 dropped out ( $4 \%$ )

Music choice based on the age of

residents

Exs focused on upper and lower body

strengthening, balance, trunk

movements,

and flexibility training

Group session

11. Venturelli et al (2011) Italy ${ }^{21}$

25 (84\% completed) RCT

4 dropped out (16\%)
Collective exs program,

Then walked as fast to reach

moderate breathlessness

training at predetermined

Exs not specified, only stated it

Routine met

No exs or specific

OT

behavior Mx training

(

$60 \mathrm{~min}$

$\times 2 \mathrm{wk}$

26 wks and

(Except during endpoint

OTs absence

$52 \mathrm{wk}$

activity)

Katz Index of ADLS

$6 \mathrm{~m}$ walk speed

Get-up-and-go test

Abnormal 1-leg

balance test

NPI

MADRS

MNA

$\begin{array}{cll}\begin{array}{l}\text { Usual care } \\ \text { provided at } \mathrm{NH}\end{array} & \text { Nurse } & 30 \mathrm{~min} \\ & \text { researcher and } & \times 2 \mathrm{wk} \\ 2 \text { research } & 4 \mathrm{wk} \\ & \text { assistants } & \end{array}$

\section{Usual care}

provided at $\mathrm{NH}$

Therapist (type
of therapist
not stated)

$30 \mathrm{~min}$
daily

not stated)

$12 \mathrm{wk}$ Started with $1-2$ min informal chatting
Walked up and down hallway (60 $\mathrm{m}$ long)

Aim to maintain constant walking speed

Moderate intensity

Completed between 3 and $5 \mathrm{PM}$

Offered cookies after sessio

Individual session
Usual activities

provided by $\mathrm{NH}$

$\begin{array}{cc}\text { Caregiver } & \text { minimum } \\ \text { trained by PT } & 30 \mathrm{~min} \\ & \times 4 \mathrm{wk} \\ & 24 \mathrm{wk}\end{array}$

Ax at baseline,

2 wks and endpoint

Modified CMAI

Ax at baseline, after $6 \mathrm{wks}$ of intervention and endpoint

MMSE

ADS 6 abbreviated

BOP

Ax at baseline and endpoint

6MWT

MMSE
Moderate exs significantly slows (by 1/3) progressive deterioration in ability to perform ADLS in individuals with

$\mathrm{AD}$ in $\mathrm{NH}$

$P$ value $<.05$, (SD)

Significant findings

Mean Katz Index of ADLs; IG $3.2(1.3)$

to $27(1.4)$ to $2.6(1.5)$ : CG $3.1(1.3)$

to $2.6(1.5)$ to $2.2(1.5)$

Mean walking speed; IG $0.33(0.14)$

to $0.41(0.17)$ to $0.41(0.16)$; CG 0.33

(0.14) to $0.37(0.17)$ to $0.36(0.19)$

Group music with movement can be

beneficial in managing agitated

behaviors in individuals with a dementia

$P$ value 0 (SD)

Significant findings

$$
\text { to } 3.9 \text { (2) to } 3.4(1.3)
$$

Music-based exs could improve cognition for individuals with a dementia Pvalue 0.05 , (SD)

Significant findings

Mean MMSE; IG 12.9 (5) to $14.4(4.4)$

to 15.5 (4.4); CG 10.8 (5) to 11.5 (5.2

to 11 (4.3)

Median ADS 6; category fluency- IG 10 to 13 to 14 , CG 6.5 to 7 to 7

Median BOP; need for help- CG 23 to 17.5 to 21

Walking program in NH with family can stabilize, for a short time, progressive, stabilize, for a short time, progressive, ADLs in advanced $A D$

$P$ value $<.05$, (SD)

$P$ value $<.05$, (SD)
Significant findings

Mean 6MWT; IG 245 (31) to 294 (49);

CG 238 (47) to 168 (34)

Mean BI; IG 34 (4) to 42 (4)

Mean MMSE; CG 12 (2) to 6 (2)

(continued on next page) 
Table 2 (continued)

\begin{tabular}{|c|c|c|c|c|c|c|c|}
\hline $\begin{array}{l}\mathrm{N}(\% \\
\text { Completing } \\
\text { Study) and } \\
\text { Drop outs (\%) }\end{array}$ & $\begin{array}{l}\text { Study } \\
\text { Design }\end{array}$ & Intervention Group (IG) & Control Group (CG) & $\begin{array}{l}\text { Intervention } \\
\text { Conducted } \\
\text { By }\end{array}$ & $\begin{array}{l}\text { Length and } \\
\text { Frequency of } \\
\text { Session and } \\
\text { Duration of } \\
\text { Study }\end{array}$ & $\begin{array}{l}\text { Outcome } \\
\text { Measures }\end{array}$ & Significant Findings \\
\hline $\begin{array}{l}\text { 12a. Williams et al (2C } \\
116 \text { (76\% completed) } \\
26 \text { dropped out }(24 \%) \\
8 \text { NH sites } \\
\text { b. Sub-set } \\
45 \text { ( } 80 \% \text { completed) } \\
9 \text { dropped out (20\%) } \\
\\
\text { c. Sub-set } \\
105 \text { ( } 78 \% \text { completed) } \\
23 \text { dropped out ( } 22 \%)\end{array}$ & $\begin{array}{l}\text { 07) USA, } \\
\text { RCT }\end{array}$ & $\begin{array}{l}\text { W. Williams et al (2008) USA, }{ }^{22} 12 \mathrm{c} \text {. } \\
\text { Individual session } \\
\text { Comprehensive exs group (EG) } \\
\text { Designed around familiar } \\
\text { functional activities } \\
\text { Strength, flexibility. } \\
\text { balance and endurance } \\
\text { exs and walking } \\
\text { Reps progressed from } 3 \text { up to } 9 \\
\text { Amount of support decreased } \\
\text { and resistance } \\
\text { increased as progressed } \\
\text { Supervised walking group (WG) } \\
\text { Walk at own pace } \\
\text { Rest and assistance PRN } \\
\text { Pace, distance and duration } \\
\text { increased incrementally } \\
\text { 1x assist walked along side } \\
\text { using a gait belt PRN }\end{array}$ & $\begin{array}{l}\text { al }(2011) \text { USA }^{14} \\
\text { Social conversation } \\
\text { Individual } \\
\text { Casual conversation } \\
\text { In own room or nearby } \\
\text { quiet room } \\
\text { Talk about topics that } \\
\text { interested them } \\
\text { Reminiscence, life } \\
\text { review and cognitive } \\
\text { exs avoided }\end{array}$ & $\begin{array}{l}\text { PT and } \\
\text { Nursing } \\
\text { students } \\
\text { trained by } \\
\text { the investigators }\end{array}$ & $\begin{array}{l}\text { WG } \\
15-30 \mathrm{~min} \\
\\
\text { CG } \\
15-30 \mathrm{~min} \\
\text { All } \times 5 \mathrm{wk} \\
16 \mathrm{wk}\end{array}$ & $\begin{array}{l}\text { Ax at baseline } \\
\text { and endpoint } \\
\text { OAS } \\
\text { AMS } \\
\text { DMAS } \\
\text { b. Sub-set } \\
\text { CSDD } \\
\text { DMAS } \\
\text { AMS } \\
\text { OAS } \\
\text { c. Subset } \\
\text { ACIF } \\
\text { 6MWT }\end{array}$ & 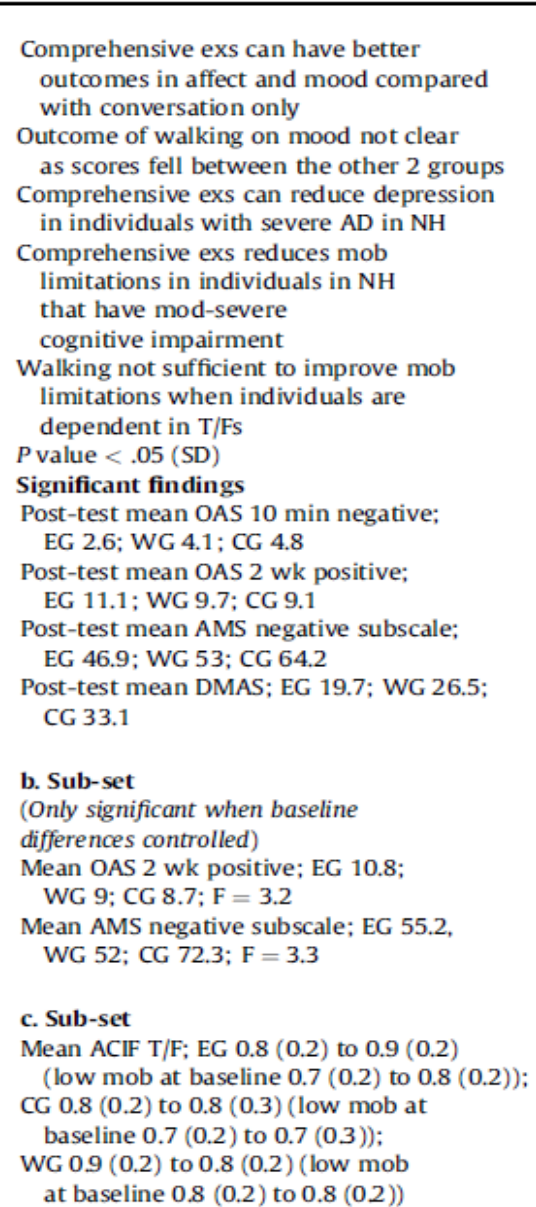 \\
\hline
\end{tabular}

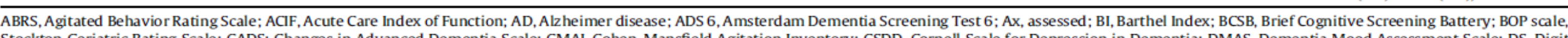

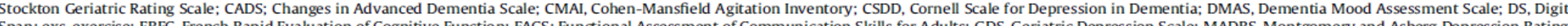

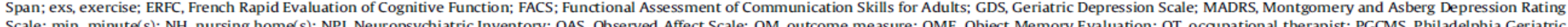

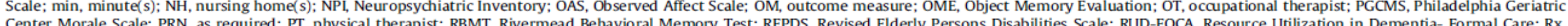

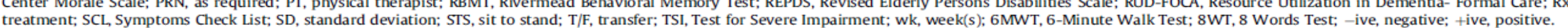




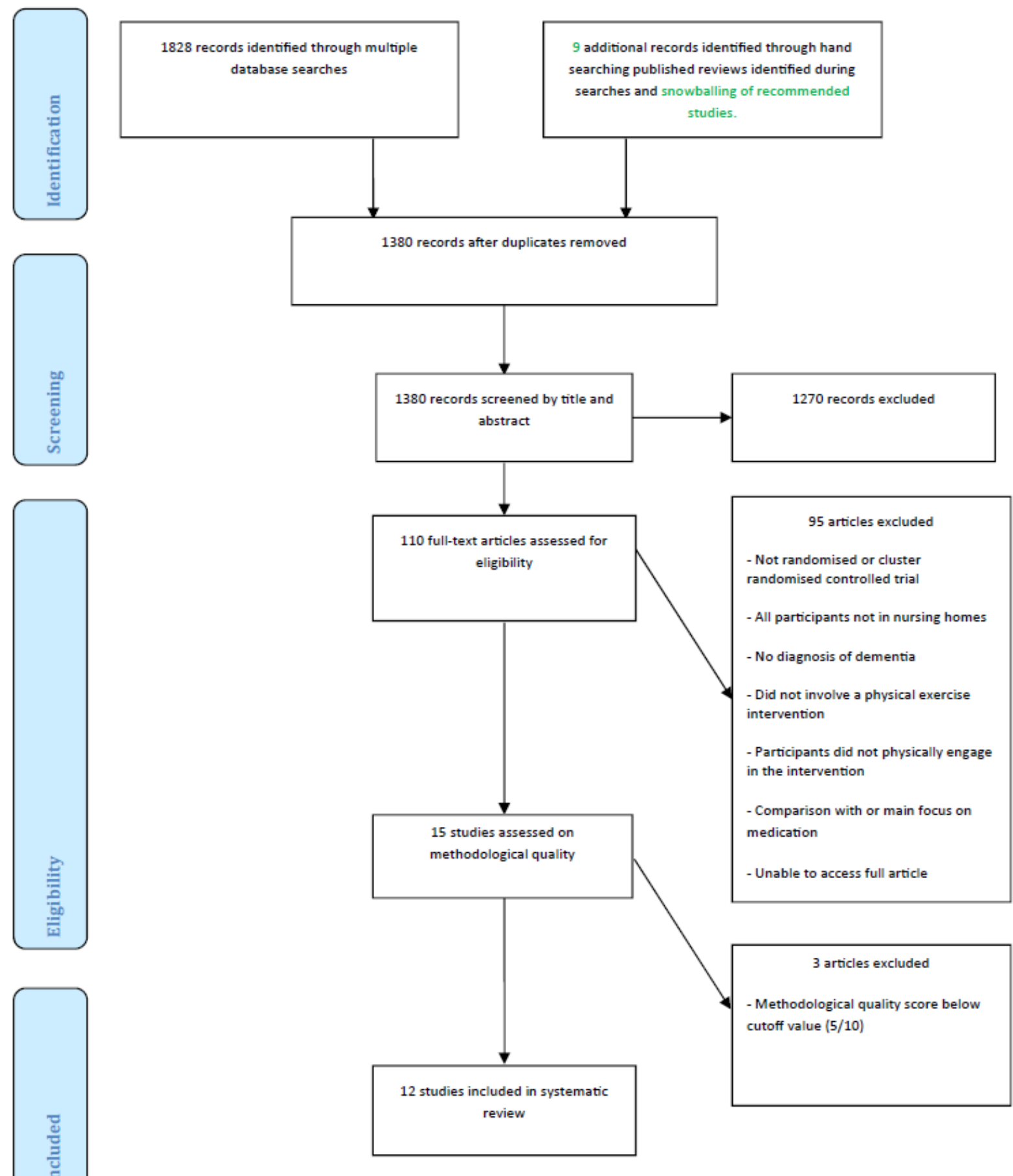

Fig. 1. Preferred Reporting Items for Systematic Reviews and Meta-Analyses flow diagram of studies through the systematic review. 